\title{
Distinctive Neuropathologic Alterations in the Deep Layers of the Parietal Cortex after Moderate Ischemic-Hypoxic Injury in the P3 Immature Rat Brain
}

\author{
STÉPHANE V. SIZONENKO, JOZSEF Z. KISS, TERRIE INDER, PETER D. GLUCKMAN, AND \\ CHRIS E. WILLIAMS
}

Unité de Dévelopement [S.V.S.], Department of Pediatrics, School of Medicine, University of Geneva, 1211 Geneva, Switzerland; The Liggins Institute [S.V.S., P.D.G., C.E.W.], School of Medicine, University of Auckland, Auckland 92019, New Zealand; Department of Fundamental Neurosciences [J.Z.K.], School

of Medicine, University of Geneva, 1211 Geneva, Switzerland; and Murdoch and Childrens Research

Institute [T.I.], Howard Florey Institute, Royal Women's Hospital, 3053 Victoria, Melbourne, Australia

\begin{abstract}
ABST
Moderate focal brain hypoxic-ischemic (HI) injury in the
immature P3 rat leads to loss of cortical volume and disruptions
of cortical myelination. In this study, we characterized the time
course and pattern of cellular degeneration, axonal disruption,
astrogliosis, and microglia activation. After moderate transient
unilateral hypoxia-ischemia, brains were collected at set time
points and positive staining was assessed. Cellular degeneration
stained with Fluoro-Jade B (FJ-B) was distributed in a columnar
pattern, primarily within the deep cortical layers V-VII extend-
ing up to layer IV of the parietal cortex (pCx). FJ-B staining
increased in the ipsilateral pCx 12 and 24 h $(p<0.05)$ after the
injury. $\beta$-Amyloid precursor protein immunoreactivity indicating
axonal disruption increased at $24 \mathrm{~h}(p<0.05)$ and showed the
same distribution as FJ-B. Glial fibrillary acidic protein-positive
astrocytes increased dramatically within the ipsilateral pCx from
$24 \mathrm{~h}(p<0.05)$ to $18 \mathrm{~d}(p<0.001)$ after HI injury and displayed
a columnar pattern extending from the deep cortical layers to
layers IV. Isolectin-B4 and ED1-labeled microglia were also
\end{abstract}
Approximately $5-10 \%$ of infants who are born at $<1500 \mathrm{~g}$ show severe spastic motor deficit, and an additional 25-50\% exhibit less severe neurodevelopmental disabilities involving motor, cognition, and behavioral deficits (1). Neuropathologic features in the premature infant include the distinct cystic white matter lesions termed periventricular leukomalacia (PVL) and, commonly, a more subtle and diffuse lesion with loss of white

Received October 3, 2003; accepted September 29, 2004.

Correspondence: Stéphane Sizonenko, M.D., Ph.D., Unité de Dévelopement, Department of Paediatrics, 6 rue Willy Donzé, 1211 Geneva 4, Switzerland; e-mail: stephane. sizonenko@medecine.unige.ch.

This work was supported by grants from the Health Research Council of New Zealand; from the New Zealand Lotteries Board; and the De Reuter, Schmidheiny and Boninchi Foundations, Geneva, Switzerland.

DOI: 10.1203/01.PDR.0000157673.36848.67 increased within the ipsilateral deep $\mathrm{pCx}$ and underlying white matter between 12 and $24 \mathrm{~h}(p<0.01)$, and increased Isolectin-B4 lasted up to $7 \mathrm{~d}$ after injury. These observations are consistent with the hypothesis that neuronal loss, astrogliosis, and microglia activation precede the subsequent disruption of cortical growth and myelination. This model offers new possibilities for investigating the cellular and molecular mechanisms of damage and repair after neonatal HI injury. (Pediatr Res 57: 865-872, 2005)
Abbreviations
$\boldsymbol{\beta}$-APP, $\beta$-amyloid precursor protein
GFAP, glial fibrillary acidic protein
HI, hypoxic-ischemic
FJ-B, Fluoro-Jade B
pCx, parietal cortex
PVL, periventricular leukomalacia

matter volume and ventriculomegaly $(1,2)$. Magnetic resonance imaging in preterm infants has revealed abnormal diffusion signal within the white matter of preterm infants with subsequent PVL (3) and also alterations in cerebral grey and white matter development (4,5). Most animal models of hypoxia-ischemia in the immature brain are pathologically relevant to the mildly preterm infant (30-34 wk) and term infants with neuronal loss in the cortex and deeper nuclear regions, including striatum, thalamus, and hippocampus (6,7). Models that closely resemble the alterations of brain development with loss of grey matter and diffuse white matter injury with ventriculomegaly seen more frequently in the more preterm infant are less available. The P3 rat shares some similarities in terms of cortical neuronal, glial, and oligodendroglial development to 
the very preterm infant $(8,9)$. To define the nature of diffuse injuries and potentially subsequent altered brain development of the very preterm infant ( $24-28 \mathrm{wk})$, we characterized the effects of a moderate unilateral hypoxic ischemic injury in the immature P3 rat brain. A moderate hypoxia-ischemia at P3 showed a reduction of cortical size with alteration in the deep cortical myelination pattern at P21 (10). The histopathologic changes underlying these alterations of cortical development after hypoxic-ischemic (HI) injury in the very immature brain were not defined. The aim of this study was to characterize the neuropathologic alterations and the time course of neuronal degeneration, axonal disruption, astrocytic, and microglial activation after the moderate unilateral $\mathrm{HI}$ injury at P3.

\section{METHODS}

The University of Auckland Ethics Committee approved these studies.

Hypoxia-ischemia. P3 Wistar pups had right carotid cauterization under halothane anesthesia. After a 30 -min recovery period at $37^{\circ} \mathrm{C}$ in $85 \pm 5 \%$ humidity, they underwent $6 \% \mathrm{O}_{2}$ hypoxia for $30 \mathrm{~min}$ in the same thermoneutral conditions. The pups then were returned to the dam and maintained in normal holding conditions at $22^{\circ} \mathrm{C}$ with a light/dark cycle of $12 \mathrm{~h}$. At the preset time points, pups were taken for brain collection.

Histologic preparation. The pups were killed with an overdose of pentobarbital and had an intracardiac perfusion of $\mathrm{NaCl} 0.9 \%$ followed by fixation of the tissue with $4 \%$ paraformaldehyde in $0.1 \%$ PBS. The brain was collected and processed in increasing percentage of ethanol and embedded in paraffin. Contiguous thin 4- $\mu \mathrm{m}$ sections at the level of the dorsal hippocampus were cut on a microtome (Leica, Germany) and collected on Polysine (Esco, Erie Scientific Co., Portsmouth, NH) slides.

Fluoro-Jade B staining. Fluoro-Jade B (FJ-B; Histo-Chem Inc., Jefferson, AR) stains for degenerating neurons and axons in a number of neuronal injury models (11) and was used in this study to assess neuronal and axonal degeneration after HI injury. The sections were co-stained with 4',6'diamidino-2-phenyllindole (DAPI; Sigma Chemical Co., St. Louis, MO) a fluorescent nuclear stain. Four-micron sections at the level of the dorsal hippocampus were cut, collected on Polysine slides, and dried overnight. They were dewaxed in xylene and hydrated. Then, following the protocol used by Schmued and Hopkins (11), the sections were co-stained with FJ-B and DAPI. After $20 \mathrm{~min}$ in the staining solution, the slides were rinsed in water washes. Excess water was removed and the slides were dried, cleared by immersion in xylene, and coverslipped with DPX (Sigma Chemical Co.) mounting medium.

Immunostaining. All immunostaining was performed using the following protocol: paraffin sections, $4 \mu \mathrm{m}$ thick adjacent to the sections used for the FJ-B staining, were collected to Polysine slides, dewaxed in xylene, and brought to water through graded alcohols. Slides were placed in a $0.1-\mathrm{M}$ phosphate buffer saline. For ED1 immunostaining, antigen unmasking was performed by incubating the sections in $0.1 \%$ trypsin (Type 2; Sigma Chemical

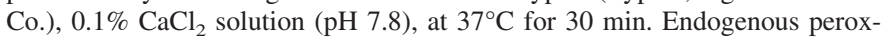
idase was blocked for $30 \mathrm{~min}$ in $0.3 \% \mathrm{H}_{2} \mathrm{O}_{2}$ in methanol. Nonspecific binding was blocked by incubating the slides in PBS buffer $1.5 \%$ horse serum [ $\beta$-amyloid precursor protein $(\beta$-APP) $]$ or $2.5 \%$ goat [glial fibrillary acidic protein (GFAP), ED1] and 1\% BSA for $30 \mathrm{~min}$ at room temperature. They then were incubated with the various primary antibodies: 1 ) to the $\beta$-APP (MAB 343 , Chemicon, Temecula, CA) diluted to $1: 100$ in $1 \%$ normal horse serum/ $0.1 \%$ BSA (Sigma Chemical Co.) in $0.1 \mathrm{M}$ Tris-HCl buffer $\mathrm{pH} 7.4-0.3 \%$ triton overnight at $4^{\circ} \mathrm{C}$; 2) to GFAP 1:400 (Z334; Dako, Glostrup, Denmark) in PBS-T overnight at $4^{\circ} \mathrm{C}$; and 3) to ED1, 1:100 (CBL1511; Cymbus Biotech, Flanders, NJ) in PBS-T overnight at $4^{\circ} \mathrm{C}$. This was followed with a $60-\mathrm{min}$ incubation at room temperature with biotinylated secondary horse anti-mouse IgG 1:200 (Vectastain kit; Vector Laboratories, Burlingame, CA), biotinylated secondary goat anti-rabbit IgG 1:400 (Life Science, Amersham, Piscataway, $\mathrm{NJ}$ ), and biotinylated secondary goat anti-mouse IgG 1:200 (Life Science, Amersham) for each of the three primary antibodies. With the use of the Vectastain $\mathrm{ABC}$ system, the sections were developed with diaminobenzidine (Sigma Chemical Co.) according to the manufacturer's instructions. For Isolectin-B4 (Molecular Probes, Eugene, OR) staining, endogenous peroxidase was blocked in $0.3 \% \mathrm{H}_{2} \mathrm{O}_{2}$ in methanol for $30 \mathrm{~min}$, and sections were incubated overnight at $4{ }^{\circ} \mathrm{C}$ with biotinylated Isolectin-B4 and revealed using the Vectastain $\mathrm{ABC}$ system and diaminobenzidine according to the manufacturer's instructions. Sections were lightly counterstained with hematoxylin-eosin, dehydrated, cleared, and mounted using Micromount (Surgipath, Richmond,
IL). In every experiment, a control for which the primary antibody was omitted was used as a negative control.

Experimental design and analysis. For characterizing the pathogenesis of the lesions after HI, pups were killed at different time points after the HI injury: $3 \mathrm{~h}(n=13), 6 \mathrm{~h}(n=13), 12 \mathrm{~h}(n=15), 24 \mathrm{~h}(n=14), 72 \mathrm{~h}(\mathrm{P} 6 ; n=14)$, $7 \mathrm{~d}(\mathrm{P} 10 ; n=13), 11 \mathrm{~d}(\mathrm{P} 14 ; n=15)$, and $18 \mathrm{~d}(\mathrm{P} 21 ; n=14)$. For minimizing external variability, a block design was used and pups in each experimental litter were taken at the different time points. Two pups from each litter remained with the dam during surgery and hypoxia of the others; these were used as control animals at the different time points. As previously shown, after $\mathrm{HI}$ at $\mathrm{P} 3$, there was no significant difference in the reduction of cortical size at P21 measured at anterior, middle, and posterior levels of the brain (striatum, dorsal hippocampus, and ventral hippocampus, respectively) (10). Therefore, in this study, we analyzed the cortex, hippocampus, thalamus, and corpus callosum (CC) at the level of the dorsal hippocampus only (Fig. 1). For each brain and staining, four contiguous sections that contained full and symmetric dorsal hippocampus were assessed. During the assessment of the brains, the investigator was blinded to the grouping of the animals.

$F J-B$ staining, $\beta-A P P, G F A P$, Isolectin-B4, and ED1 immunoreactivity assessment. As described by Schmued and Hopkins (11), FJ-B is an anionic fluorescein derivative used for positive staining of degenerating neurons and axons. The high signal-to-noise ratio means that fine neuronal processes, including distal dendrites, axons, and axon terminals, can be more readily detected. DAPI is a fluorescent nuclear stain. $\beta$-APP is a protein found in the neuronal cell and is associated with the cytoskeleton's being transported by fast anterograde axonal transport (12). $\beta$-APP accumulation is a sensitive marker of axonal injury and dysfunction in traumatic (13), ischemic (14), or chemical (15) injury. In this study, the accumulation of $\beta$-APP immunostaining in cell bodies and axons with retracted processes was considered as positive for axonal disruption. GFAP is an intermediate filament that is up-regulated in response to injury to the CNS and was used as a marker for glial reactivity in the brain. The Griffonia simplicifolia Isolectin-B4 selectively recognizes $\alpha$-D-galactosyl residues contained in membrane glycoconjugates, and histochemical methods based on incubation of brain tissue with this lectin label microglia cells forms, macrophages, and microvasculature (16). Isolectin-B4 has been used to study rat brain microglia development $(17,18)$ as well as microglia activation after brain injury $(19,20)$. ED1 antibody recognizes activated microglia-macrophages and is considered as a marker of microglia activation $(19,21,22)$.

For histopathologic assessment, sections at the level of the dorsal hippocampus were evaluated for positive staining with FJ-B under fluorescent microscope with a blue excitation light $(450-490 \mathrm{~nm})$ and with a UV excitation (330-380 nm) for DAPI. $\beta$-APP, GFAP, Isolectin-B4, and ED1 immunostainings were assessed under light microscopy. Fluorescence and immunoreactivity were assessed in the area between the rhinal sulcus laterally, the cingulum medially, and the external boundaries of the $\mathrm{CC}$ [parietal cortex $(\mathrm{pCx})$ in Fig. 1]. This area includes the motor and somatosensory cortices and

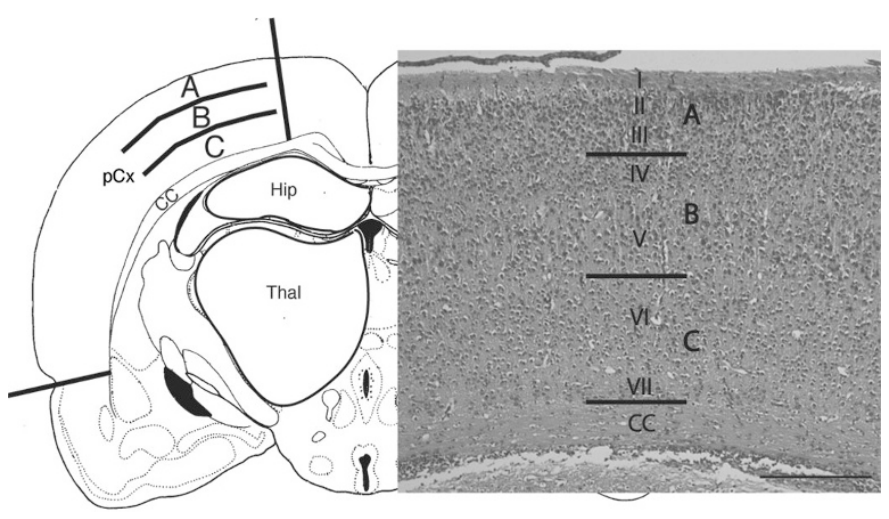

Figure 1. Areas of the brain scored for FJ-B, $\beta$-APP, GFAP, Isolectin-B4, and ED1. (Left) On sections at the level of the dorsal hippocampus, the $\mathrm{pCx}$ was defined as the cortical area between the cingulum medially and the rhinal sulcus laterally. The $\mathrm{pCx}$ was divided in superficial band (A), middle band (B), and deep band (C). The hippocampus (Hip), thalamus (Thal), and the underlying CC were also scored. (Right) Each of the bands scored (A, B, and C) corresponds to a particular cortical region at $\mathrm{P} 3$ : A and $\mathrm{B}$ are the developing layers I-III and IV-V of the cortex, respectively, and are composed of the more immature neurons, and $\mathrm{C}$ corresponds to layers VI and VII. This later corresponds to the remaining subplate. Bar $=500 \mu \mathrm{m}$. 
the entorhinal cortex. It is referred to as the $\mathrm{pCx}$ in the rest of the text. Hippocampus, striatum, and CC were also investigated. No degenerating neurons were seen in these later regions, and no further analysis was performed except for GFAP, Isolectin-B4 and ED1 in the CC. A previously published semiquantitative method (23) was used to assess for the ranges of positive fluorescent/immunopositive cells observed: no positive cells, $0 \%$; scattered positive cells, $<10 \% ; 10-50 \%$ of positive cells; $50-90 \%$ of positive cells; and $>90 \%$ of positive cells. Each defined region of the cortex was scored according to the range of fluorescent or immunopositive cells seen. For statistical analysis, the ranges attributed by the examiner were converted to a ratio scale of degenerating cells by taking the nominal cellular degeneration as the midpoint of each of these ranges $(0,5,30,70$, and $95 \%$, respectively) as previously described (23). The extent of FJ-B fluorescence and $\beta$-APP, GFAP, Isolectin-B4, and ED1 immunopositivity in the $\mathrm{pCx}$ was calculated as the mean $\pm \mathrm{SD}$ of the percentage of fluorescence and immunopositivity for the $\mathrm{pCx}$ area from each slide. For assessing which cortical depth was most vulnerable to injury, $\mathrm{pCx}$ was divided further into three superficial, middle, and deep cortical bands corresponding anatomically, at the time of injury (P3), to layers I-III, layers IV-V, and layer VI plus remaining subplate layer VII (Fig. 1). The remaining subplate was localized at the base of the cortical plate, immediately below layer VI and above the CC. Bands A, B, and C were determined in the characteristic seven-layered neocortex, with A containing the outer layers I-III, B containing layers IV-V, and C containing the deeper layers VI-VII (Fig. 1). The extent of damage in the three levels of the $\mathrm{pCx}$ were scored and calculated as the mean $\pm \mathrm{SD}$ of the percentage of fluorescence and immunoreactivity for each band (A, B, C) from the four sections. As the data are not absolutely continuous, comparisons of the percentage of positive cells for FJ-B, $\beta$-APP, GFAP, Isolectin-B4, and ED1 in the ipsilateral and the contralateral hemispheres for each of the time points were made with the nonparametric Wilcoxon sign rank test and expressed as mean $\pm \mathrm{SD}$. A $p<0.05$ was considered as significant.

\section{RESULTS}

Cell injury stained with FJ-B. FJ-B-positive fluorescent cells were distributed in the $\mathrm{pCx}$ between the cingulum and rhinal sulcus (Fig. 2). No labeled cells were seen in the other regions ipsilaterally or in the contralateral hemisphere. In the contralateral side, rare FJ-B-positive cells could be seen (Fig. $2 A$ ). In the ipsilateral $\mathrm{pCx}, \mathrm{FJ}-\mathrm{B}$ revealed different patterns of cell degeneration. Cellular degeneration appeared scattered (Fig. 2B), columnar (Fig. 2C), or columnar with confluence in the deep layers of the cortex (Fig. 2D). The degenerating cells in columns were extending from the deep cortical layers (band C) into the middle layers (band B) and up to the deeper limit of the external layers (band A). In the most severely damaged cortex, the lesion was extending sidewise, leading to confluence in the deeper layers rather than extending into the external layers (Fig. 2D). The external cortical layers I-III (band A) were injured in $11(10 \%)$ of the 111 animals studied, and these were the more severely injured animals. In contrast, the middle layers IV-V (band B) were injured in the less severely damaged animals [25 of $111(22.5 \%)$ ]. The majority of the injury was identified in the deep layers VI and VII (band C). At higher magnification, degenerating neurons that retained their initial pyramidal shape were seen in the deep and middle cortical layers IV-VII. Often these cells displayed enlarged processes with varicosities (Fig. 2E). DAPI staining revealed viable cell nuclei as blue structures (Fig. $2 F$ ). Cells with intense light blue to white fluorescence were distributed in a columnar pattern that corresponded with FJ-B-positive degenerating cells (Fig. $2 E$ and $F$ ). At high magnification, these cells when examined under FJ-B excitation light appeared condensed (Fig. 2G), with DAPI staining showing bright round nuclear structures, suggestive of condensed chromatin clumps
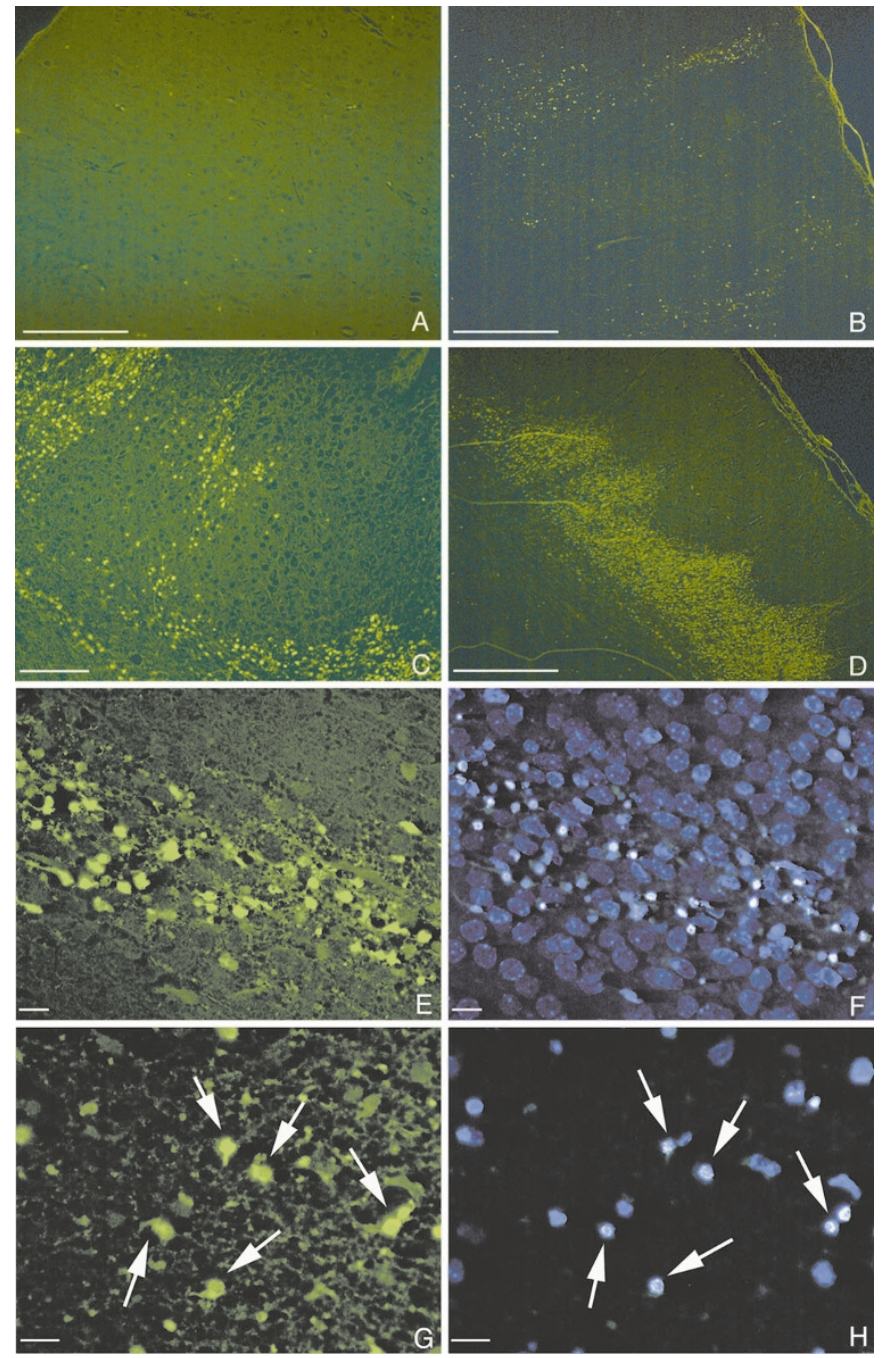

Figure 2. Distribution of degenerating cells. $(A-D)$ Degenerating cells $24 \mathrm{~h}$ after $\mathrm{HI}$ insult stained with FJ-B. (A) Contralateral cortex: rare fluorescent cells were observed. Bar $=500 \mu \mathrm{m}$. $(B)$ Ipsilateral cortex: scattered fluorescent cells were observed in a columnar pattern. Bar $=500 \mu \mathrm{m}$. $(C)$ Ipsilateral cortex, moderate damage: a columnar aspect of positive cells was present. Bar $=100 \mu \mathrm{m}$. $(D)$ Ipsilateral cortex, severe damage: a columnar aspect of positive cells was present with confluence of positive cells in the deep layer. Bar $=500$ $\mu \mathrm{m}$. $(E-H)$ FJ-B and DAPI staining $24 \mathrm{~h}$ after injury. The same area was photographed under blue excitation for FJ-B $(E$ and $G)$ and UV excitation for DAPI visualization $(F$ and $H)$. ( $E$ and $G$ ) High magnification of degenerating neurons. Axonal degeneration (arrows) and varicose swelling of the axons are visible. $(E$ and $F$ ) Some FJ-B-positive degenerating cells show an intense and bright signal with DAPI staining. ( $G$ and $H$ ) At higher magnification, this intense white signal appears as condensed nuclear structures in degenerating neurons (arrows). Bar $=10 \mu \mathrm{m}$.

similar to that found in apoptotic delayed cell death (Fig. $2 \mathrm{H}$ ) (24).

We observed a substantial increase in the number of FJ-Bpositive cells at $12 \mathrm{~h}$ after injury $(p<0.05)$. The peak of cellular degeneration was reached at $24 \mathrm{~h}(p<0.01)$ and decreased below significance at $72 \mathrm{~h}$ when compared with the contralateral pCx (Fig. 3A). From $48 \mathrm{~h}$ to $18 \mathrm{~d}$ (P21), a nonsignificant level of degenerating cells remained. The external, middle, and deep bands of the $\mathrm{pCx}$ were assessed separately to determine which layers were the most vulnerable to HI injury (Fig. 3B). Significantly increased FJ-B-positive cells 


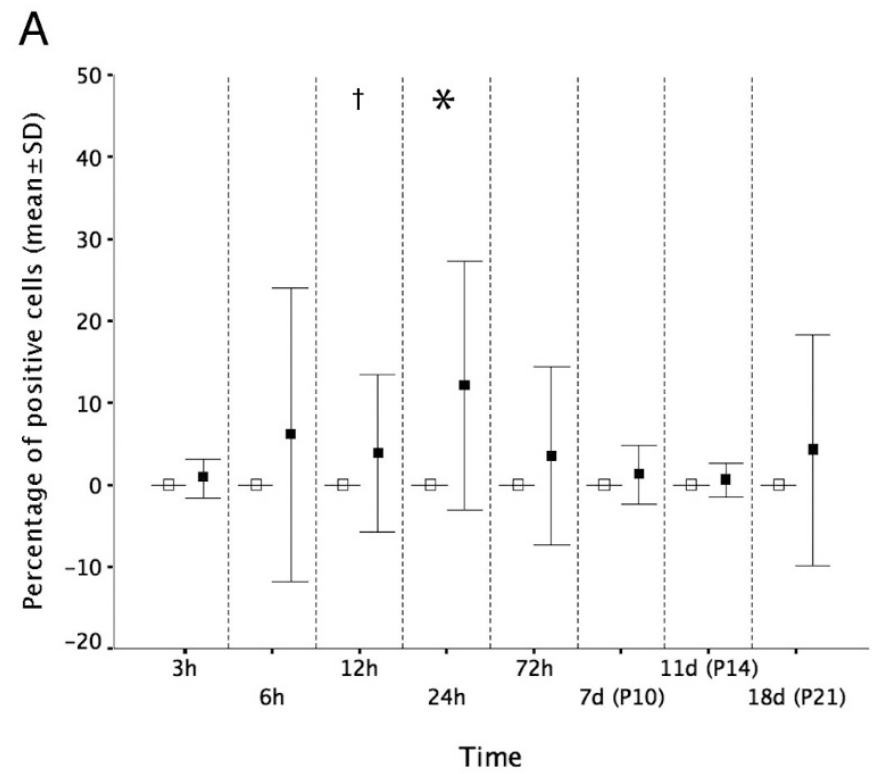

B

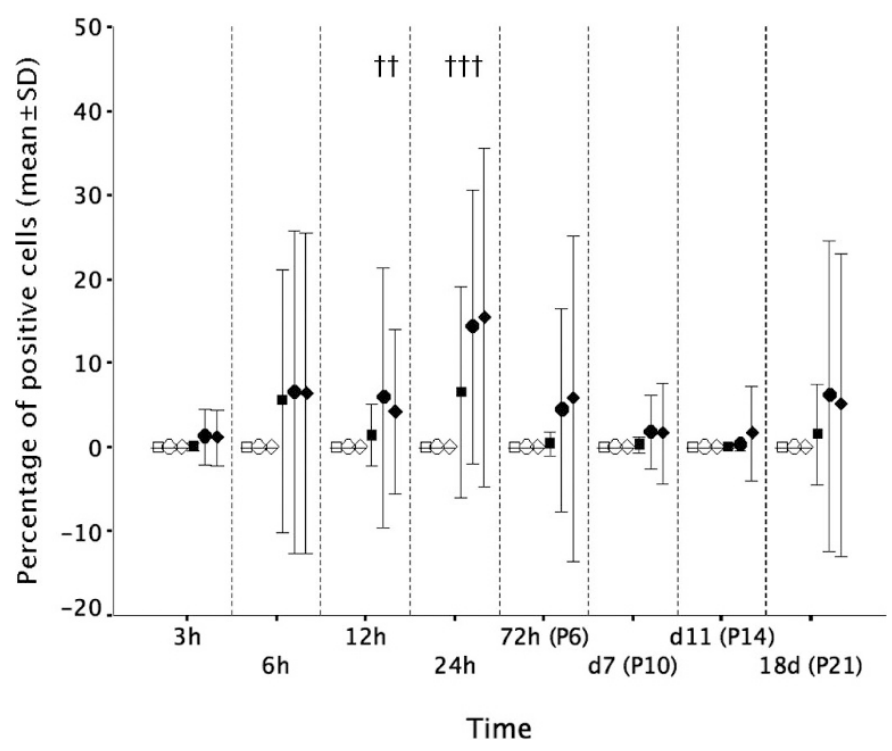

Figure 3. Time course of cellular degeneration after $\mathrm{HI}$ injury in the $\mathrm{P} 3$ rat brain. $(A$ and $B$ ) At each time point, brains from injured animals were stained with FJ-B. Cells showing green fluorescence were scored. (A) Data are shown as mean \pm SD of the scores of the contralateral $\mathrm{pCx}(\square)$ and of the ipsilateral pCx ( $\square$ ) for each time point. $\dagger p<0.05 ; * p<0.01$. (B) Score of cellular degeneration in the external (A), middle (B), and deep bands (C) of the $\mathrm{pCx}$. The deep and middle layers seemed to be sensitive to hypoxia-ischemia, whereas the external layers remained mostly unaffected. Data are shown as mean $\pm \mathrm{SD}$ of the scores for each cortical depth of the contralateral $\mathrm{pCx}$ (open symbols) and of the ipsilateral $\mathrm{pCx}$ (filled symbols) at each time point. Square, external band (A); circle, middle band (B); diamond, deep band (C). $\dagger p<0.05$.

were present in the middle and deep layers at $12 \mathrm{~h}(p<0.05)$ and in all three layers $24 \mathrm{~h}$ after the injury $(p<0.05)$. No significant staining was present at any time point in the contralateral $\mathrm{pCx}$ and in control animals.

Axonal injury stained with $\beta$-APP immunostaining. Only cells in the ipsilateral $\mathrm{pCx}$ showed accumulation of $\beta$-APP immunostaining. No accumulation of $\beta$-APP was seen in cells in the other regions ipsilaterally and in the contralateral hemi- sphere. The pattern of $\beta$-APP staining was similar to that of FJ-B (Fig. $4 A$ and $B$ ). Columns of $\beta$-APP-positive cells were present in the $\mathrm{pCx}$, extending from the deep to the middle layers (Fig. 4A). No injuries were seen in the medial and lateral areas of the cortex. The outer layers (I-III) of the cortex displayed no positive staining with $\beta$-APP. Instead, a confluence of positive cells in the deeper layers (IV-VI and subplate) was seen in the more severely damaged animals. At high magnification, accumulation of $\beta$-APP could be seen in the neurons in the damaged area with a clear retraction, clubbing of the axons (Fig. 4B). No distal axons within the white matter of CC showed accumulation of $\beta$-APP.

A significant increase in the number of cells showing $\beta$-APP accumulation from $12 \mathrm{~h}$ onward was present. The peak of axonal injury was reached at $24 \mathrm{~h}(p<0.05)$ and declined at $72 \mathrm{~h}$ (Fig. $4 C$ ). Positive cells disappeared at later time points up to $21 \mathrm{~d}$ of age (18 d after hypoxia-ischemia). No labeled cells were present at any time point in the normal animals. The increase in positive cells for $\beta$-APP was significant in the middle (IV-V) and deep (VI-VII) cortical layers at $24 \mathrm{~h}(p<$ 0.05 ; Fig. $4 D$ ). A nonsignificant accumulation of $\beta$-APP was present in the deeper layers at 12 and $72 \mathrm{~h}$ after the injury.

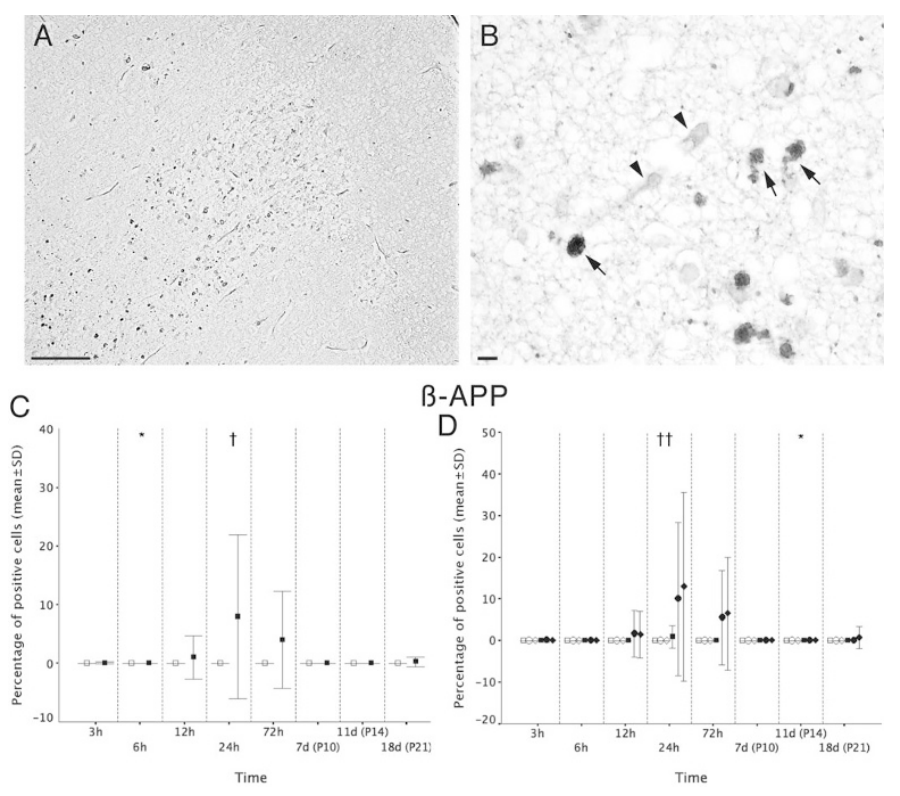

Figure 4. Pattern and time course of axonal degeneration after $\mathrm{HI}$ injury in the $\mathrm{P} 3$ rat brain. ( $A$ and $B$ ) Pattern of $\beta$-APP immunostaining $24 \mathrm{~h}$ after the injury. (A) Columnar pattern of $\beta$-APP staining with degenerating axons seen in the deep and middle layers of the cortex. Bar $=100 \mu \mathrm{m}$. $(B)$ At higher magnification, degenerating axons showed intense positive staining in the neuronal body with retraction balls and clubbing of the axon (arrows); normal neurons and axons show lighter staining (arrowheads). Bar $=10 \mu \mathrm{m}$. ( $C$ and $D$ ) Time course of positive immunostaining for $\beta$-APP in the P3 rat brain after mild $\mathrm{HI}$ injury. At each time point, brains from injured animals were stained for $\beta$-APP. The cells that showed positive staining were scored. (C) $\beta$-APP scores of the contralateral pCx $(\square)$ and of the ipsilateral pCx ( $\square$ ) at each time point. $* p<0.05$. Data are shown as mean \pm SD. (D) Score of positive $\beta$-APP immunostaining in the external (A), middle (B), and deep bands (C) of the $\mathrm{pCx}$. The deep and middle layers seemed to be the most sensitive to hypoxiaischemia, whereas the external layers remained mostly unaffected. Data are shown as mean \pm SD of the scores for each cortical depth of the contralateral $\mathrm{pCx}$ (open symbols) and of the ipsilateral pCx (filled symbols) at each time point. Square, external band (A); circle, middle band (B); diamond, deep band (C). ${ }^{*} p<0.05$. 
Reactive astrogliosis revealed with GFAP. The $\mathrm{pCx}$ and $\mathrm{CC}$ displayed strong immunostaining for GFAP. Few positive cells were seen in the other ipsilateral regions and in the contralateral hemisphere. GFAP-positive cells were also present in the $\mathrm{CC}$ underlying the $\mathrm{pCx}$. Similar to FJ-B and $\beta$-APP staining, the pattern of positive GFAP cells showed a columnar distribution within the deep and middle layers (IV-VII) of the $\mathrm{pCx}$ in the more severely damaged animals (Fig. 5A). GFAPpositive cells showed an activated astrocytic morphology with larger cell body and thicker processes (Fig. 5B). In the mildly damaged brains, the cellular distribution of GFAP staining seemed more diffuse within the deep cortical layers IV-VI.

A significant increased level of GFAP staining was present at $24 \mathrm{~h}(p<0.05)$ and remained at the later time points up to $18 \mathrm{~d}$ (P21) after the injury ( $p<0.05$; Fig. 5C). Similarly, in the ipsilateral CC, a significant increase of GFAP-immunopositive cells was present from $24 \mathrm{~h}(p<0.01)$ after the injury and persisted up to $18 \mathrm{~d}$ after the insult $(p<0.01$; Fig. $5 D)$.

Microglia activation revealed with Isolectin-B4 and EDI. Isolectin-B4-positive cells were present in both ipsilateral and contralateral cortex and in the CC in all sections analyzed. In the ipsilateral hemisphere, cell morphology differences could be seen within the deep cortical layers and the underlying CC
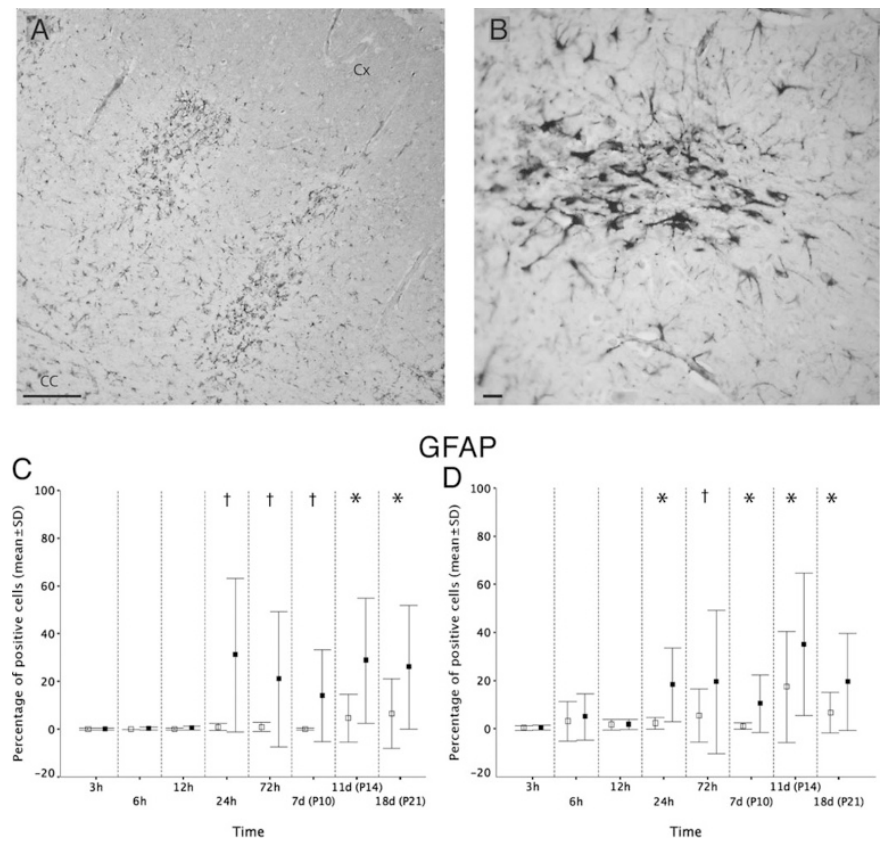

Figure 5. Pattern and time course of positive immunostaining for GFAP in the $\mathrm{pCx}$ and in the $\mathrm{CC}$ after $\mathrm{HI}$ injury in the $\mathrm{P} 3$ rat brain. ( $A$ and $B$ ) GFAP immunostaining $24 \mathrm{~h}$ after the HI injury. (A) Ipsilateral $\mathrm{pCx}$ of a severely damaged brain: dense columnar pattern of GFAP-positive astrocytes in cortical layers IV to VII is present. Bar $=100 \mu \mathrm{m}$. (B) At higher magnification, GFAP-positive astrocytes appear hypertrophic. $\mathrm{Cx}$, cortex. Bar $=10 \mu \mathrm{m}$. $(C$ and $D$ ) At each time point, cells that showed GFAP-positive staining were scored. $(C)$ Time course of positive immunostaining for GFAP in the pCx. Data are shown as mean $\pm \mathrm{SD}$ of the scores of the contralateral pCx $(\square)$ and of the ipsilateral pCx (ם). $\dagger p<0.05$; $* p<0.01$. (D) Time course of positive immunostaining for GFAP in the CC after HI injury. On the same section used to score the GFAP-positive cells in the pCx, the amount of GFAP-positive cells in the $\mathrm{CC}$ was similarly scored. Data are shown as mean $\pm \mathrm{SD}$ of the scores

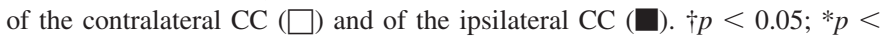
0.01 .
(Fig. 6A-D). In the injured cortex and underlying $\mathrm{CC}$, cell bodies appeared round, enlarged with reduced and thicker processes and increased staining. In the ipsilateral CC, a medial to lateral gradient of activated microglia was seen, with more round amoeboid microglia present in the medial part of the CC near the cingulum. In the contralateral cortex and $\mathrm{CC}$, with increasing postnatal age, Isolectin-B4-positive cells showed a shift from immature round cells to the morphology of resting ramified microglia with small cell body and extended thin processes. In parallel, they showed reduced staining. IsolectinB4-positive vessels were also present in both cortices.

In addition to morphology changes, the ipsilateral $\mathrm{pCx}$ Isolectin-B4 staining was increased at 12,24 , and $72 \mathrm{~h}(p<$ $0.01)$; remained high at $7 \mathrm{~d}(p<0.05)$; then decreased at the later time points (Fig. $6 E$ ) when compared with the contralateral cortex. Similar in the ipsilateral CC, an increase of Isolectin-B4-positive cells was also present at 12,24 , and $72 \mathrm{~h}(p<$ $0.01)$ and $7 \mathrm{~d}(p<0.05)$ after injury (Fig. 6F).

Whereas the ipsilateral $\mathrm{pCx}$ and $\mathrm{CC}$ showed positive immunostaining for ED1, very few positive cells were seen in the other regions scored and in the contralateral hemisphere. Similar to the pattern described for FJ-B, $\beta$-APP, GFAP, and Isolectin-B4 staining, ED1-positive cells were localized within the deep and middle layers of the $\mathrm{pCx}$ (IV-VII) and within the ipsilateral underlying CC. Positive ED1 cells appeared uniformly round within the ipsilateral deep cortex and the CC.

In the ipsilateral pCx, ED1 immunoreactivity was increased at 12 and $24 \mathrm{~h}(p<0.05)$ and then progressively decreased at the later time points (Fig. $6 G$ ). In the ipsilateral CC, an increase of ED1-positive cells was also present at 12 and $24 \mathrm{~h}(p<$ 0.05 ) after injury (Fig. 6H). In general, the overall amount of ED1-labeled microglia in the ipsilateral cortex and $\mathrm{CC}$ remained low compared with Isolectin-B4 staining of resting and activated microglia.

\section{DISCUSSION}

In our previous work, we demonstrated that a unilateral moderate hypoxia-ischemia at P3 leads to a distinctive reduction of cortical size with an altered pattern of cortical myelination $18 \mathrm{~d}$ after the HI injury (10). The aim of this study was to provide a detailed qualitative characterization of the histopathologic changes after this HI injury. In addition, these qualitative alterations were supported by a semiquantitative assessment of neuroaxonal injury and astrogliotic and microglial activations with time. However, if this model were to be used for testing of protective therapies, then precise cell count and density measurements should be performed to account for changes in brain area after hypoxia-ischemia. In this descriptive histopathologic study, we demonstrate that most neuroaxonal damage was limited to the deep layers IV-VII of the $\mathrm{pCx}$ 12-24 h after a moderate HI injury in the immature P3 rat brain. We also showed that neuronal injury is followed with a prolonged astrocytic and microglial activation in the damaged cortical area and the underlying CC. The cerebral development alterations seen in this model seem relevant to diffuse injury and altered brain development that has been shown by magnetic resonance imaging in the very preterm infant $(4,5,25)$. 

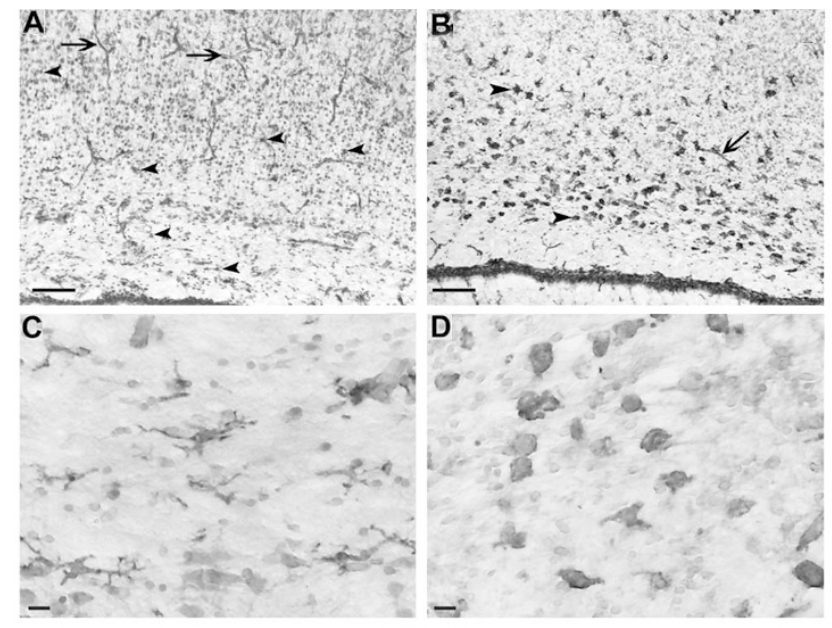

ISO-B4
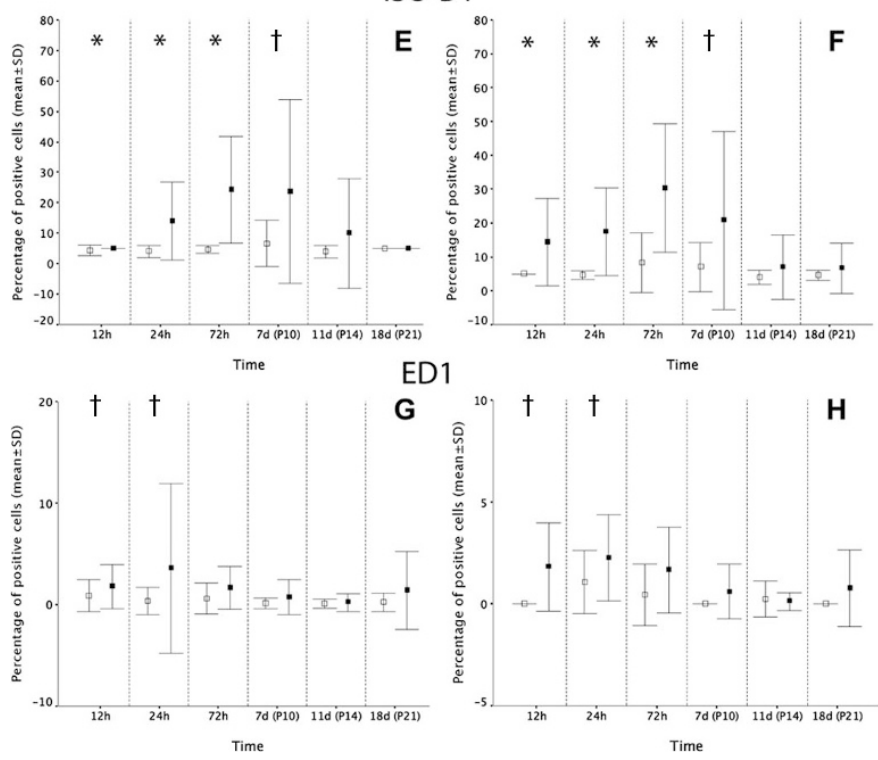

G

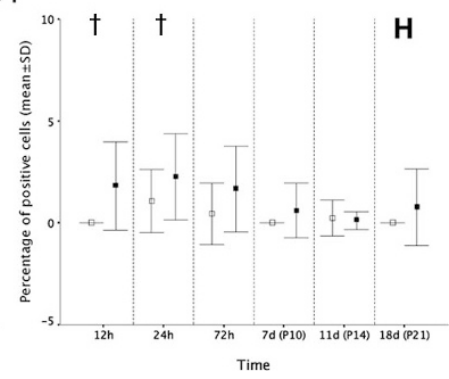

Figure 6. Pattern and time course of microglia activation in the $\mathrm{pCx}$ and in the $\mathrm{CC}$ after $\mathrm{HI}$ injury in the $\mathrm{P} 3$ rat brain. $(A-D)$ Isolectin-B4 immunostaining $24 \mathrm{~h}$ after HI. (A) Contralateral hemisphere showed disseminated positive microglia cells within the cortex and the underlying white matter (arrowheads). Vessels are also stained (arrows). Bar $=100 \mu \mathrm{m}$. (B) In the ipsilateral hemisphere, increased positive cells could be seen within the deep layers of the cortex and in the underlying white matter. These cells appeared enlarged with reduced and thicker processes (arrowheads). Bar $=100 \mu \mathrm{m}$. (C) At higher magnification, microglia in the contralateral hemisphere appeared in their characteristic resting morphology with small cellular bodies and extended thin processes. Bar $=10 \mu \mathrm{m}$. $(D)$ In the ipsilateral hemisphere, activated microglia showed enlarged cell bodies with shortening and thickening of the processes. Bar $=10 \mu \mathrm{m}$. $(E$ and $F)$ Time course of Isolectin-B4-immunopositive cells in the $\mathrm{pCx}$ and $\mathrm{CC} .(E)$ Time course of positive immunostaining for Isolectin-B4 in the pCx. Data are shown as mean \pm SD of the scores of the contralateral $\mathrm{pCx}(\diamond)$ and of the ipsilateral $\mathrm{pCx}(\diamond), \dagger p<0.05,{ }^{*} p<0.01$. (F) Time course of positive immunostaining for Isolectin-B4 in the CC. On the same section used to score the Isolectin-B4-positive cells in the $\mathrm{pCx}$, the amount of Isolectin-B4 positive cells in the CC was similarly scored. Data are shown as mean \pm SD of the scores of the contralateral CC $(\diamond)$ and of the ipsilateral CC $(\downarrow)$. $\# p<0.05, * p<0.01$. ( $G$ and $H$ ) Time course of ED1-positive cells in the $\mathrm{pCx}$ and $\mathrm{CC} .(G)$ Time course of positive immunostaining for ED1 in the pCx. Data are shown as mean \pm SD of the scores of the contralateral pCx ( $\square$ ) and of the ipsilateral pCx $(\square)$. \#p $<0.05$. (H) Time course of positive immunostaining for ED1 in the CC. On the same section used to score the ED1-positive cells in the pCx, the amount of ED1-positive cells in the CC was similarly scored. Data are shown as mean \pm SD of the scores of the contralateral CC $(\square)$ and of the ipsilateral CC ( $\square)$. $\dagger p<0.05$.
Brain immaturity and pattern of injury. The deep cortical columnar pattern of damage, with no injury in other regions, rather than laminar damage seen in older rat after hypoxiaischemia (6) is likely to be a reflection of the developmental vulnerability at this stage of cerebral maturation. The damage was predominantly in the deeper layers V-VI and subplate (layer VII) of the $\mathrm{pCx}$, with no damage in the outer layers. Several studies in rodents and sheep have shown a higher vulnerability to hypoxia-ischemia of mature neurons found in these deeper layers in comparison with immature neurons found in layers I-III $(6,26,27)$. This difference in vulnerability between immature and mature neurons is likely to be related, at least in part, to their susceptibility to excitotoxic injury $(28,29)$ and to oxidative metabolism (30). In addition, the columnar and deep cortical pattern of damage is consistent with the immaturity of the vasculature and its autoregulation at this age. Side branches between the long penetrators are not fully developed at this age, thus creating watershed regions that are more vulnerable to hypoxia $(1,31)$. Therefore, the columns of injury are likely to be found in areas where the density of the vascular system is low. Recently, McQuillen et al. (32) in a model of HI injury in P1-2 rat pups showed a similar pattern of damage: $12 \mathrm{~h}$ after hypoxia-ischemia, increased dying cells were seen in the deep cortical layers of the $\mathrm{pCx}$ at the level of the dorsal hippocampus, with other brain regions showing very limited injury. This cell death decreased at $24 \mathrm{~h}$ and was not detectable by $4 \mathrm{~d}$ after injury. It was accompanied with increased GFAP immunostaining that had the same distribution as dying cells. Furthermore, they showed a specific decrease in the number of subplate neurons. The subplate layer has been shown to assist in targeting the major axonal pathways into the cortex, including the corticothalamic and corticofugal axons during fetal brain development (33). Most of these cells then undergo apoptosis. In rodents, remaining neurons form a thin, distinct layer VII and the interstitial cells of the white matter (34). Neurons in layer VII participate in local and longdistance cortico-cortical connections but do not send projections to subcortical regions (35) and thus should be considered as part of the mature cortical neuronal mantle. Thus, injury of these deep subplate neurons during early postnatal brain development is likely to alter neuronal connectivity of the mature brain and lead to abnormal brain development and neurologic dysfunctions. Motor deficits have been shown to be present in mature rats after an HI injury at P1-2 (32).

Relevance to the diffuse component of brain injury in the preterm. This model is a moderate injury model and does not produce overt necrotic lesions compared with the P7 HI model (26) but leads to alterations of cortical development and myelination at P21 (10). Localized necrotic and/or apoptotic cell death is likely to be present, and the DAPI stain showed the presence of nuclei with features of apoptotic cell death in the columns of degenerating cells. Neurons with presumed axonal injury, demonstrated by $\beta$-APP immunostaining accumulation, showed swollen, irregular, and distended axons. These morphologic features were similar to that described in neuropathologic studies of preterm brains with diffuse brain injury $(36,37)$. The neuroaxonal damage found in the first $24 \mathrm{~h}$ may favor axonopathy as a direct effect of hypoxia-ischemia. Sim- 
ilarly, in neuropathologic studies of PVL in the preterm infant, $\beta$-APP-positive axons have been found at an early stage with involvement of pyramidal neurons from cortical layer $\mathrm{V}$, and $\beta$-APP accumulation was suggested as an early sign of neuroaxonal damage in PVL (38). Our study demonstrates that neuronal death and axonal disruption were early and prominent events after moderate HI injury in a P3 rat model. The increased and persisting astrogliosis after the acute $\mathrm{HI}$ injury was present in the area of injury in a similar pattern as seen in previous animal models (39) and in preterm infants with PVL (40). Whereas neuroaxonal degeneration occurred early after hypoxia-ischemia, astrocytes were activated during the same period and remained activated at later time points. The role of this persistent gliosis has to be determined. Astrocytic activation after CNS injury is believed to promote recovery by isolating injured regions from the rest of the brain. Furthermore, activated microglia, detected by Isolectin-B4-positive cells showing morphologic changes, was increased $72 \mathrm{~h}$ after the injury and then decreased at $7 \mathrm{~d}$ after hypoxia-ischemia. Similar to our findings, studies on microglia/macrophages activation after severe $\mathrm{HI}$ injury in the P7 rat have shown a microglia activation within $24 \mathrm{~h}$ of the injury, peaking between 48 and $96 \mathrm{~h}$ and then disappearing at later time points $(19,21,41)$. The differences in the amount of Isolectin-B4 and ED1-positive cells during the time course are likely to be a reflection of the different microglia populations labeled with these markers. Milligan et al. $(18,22)$ proposed that ED1positive cells are brain macrophages and do not represent the overall population of microglia/macrophages that are present in the brain or activated after brain injury. Thus, Isolectin-B4 stains the overall population of brain microglia/macrophages, whereas ED1 is limited to brain macrophages $(18,22,41)$. The clear morphologic modifications of Isolectin-B4-positive cells from quiescent arborized cells to round amoeboid shape that was present in the injured cortical areas and in a decreasing gradient from the medial to the lateral $\mathrm{CC}$ likely represent microglial activation but could also be recruitment of immature microglial cells that show a similar morphology $(19,42)$.

The mechanisms underlying distinctive pathologic changes after preterm brain injury and the subsequent developmental perturbation are not yet fully understood (43). Our observations suggest that, in this model, one important mechanism of damage leading to the subsequent deficit in cortical myelination previously described (10) is the early neuroaxonal injury followed by a prolonged astrogliosis and microglia activation. This does not exclude injury to the developing oligodendrocytes. It has been proposed that oligodendrocyte progenitors are particularly vulnerable to ischemia at this developmental period and that the loss of these cells leads to deficient myelination $(44,45)$. In this study, we did not address this important issue. However, in a preliminary experiment, we found an immediate and significant increase in NG2-positive oligodendrocyte progenitors after hypoxia-ischemia with no evidence of apoptotic death of these cells (46). During the postnatal period analyzed here, there is an intense gliogenesis (including oligodendrocytes, astrocytes, and microglia) with proliferation, differentiation, and migration of all cell lineages. Therefore, during these highly dynamic processes, multiple immunolabel- ing with oligodendrocyte lineage-specific markers (NG2, O4, O1, A2B5, and MBP) $(8,47)$ in combination with neurofilaments, cell death, and proliferation markers would be required for a detailed assessment of damage and recovery in the oligodendrocyte population.

\section{CONCLUSION}

In conclusion, our study documented that a moderate HI injury to the P3 rat brain leads to neuroaxonal damage in the deep cortical layers (IV-VII) during the initial 12-24 h after the injury. In addition, important astrogliotic and microglial reactions started at the same time and persisted for weeks after the initial injury. The neuroaxonal disruption and gliosis described in our model of moderate $\mathrm{HI}$ injury in the P3 immature brain are comparable to certain neuropathologic features of diffuse PVL seen in preterm infants $(40,48)$. The early neuropathologic events described in this study certainly contribute to the loss of cortical volume and disruption of myelination at P21 seen in the long term with this model (10). To define the relationship of diffuse white matter injury with altered cortical development in the preterm infant, further analysis of the cell types and of the damage and repair mechanisms involved will be necessary.

Acknowledgments. S.V.S. was the recipient of a postdoctoral fellowship from the Geneva University Hospital (Switzerland).

\section{REFERENCES}

1. Volpe JJ 2001 Neurobiology of periventricular leukomalacia in the premature infant Pediatr Res 50:553-562

2. Leviton A, Gilles F 1996 Ventriculomegaly, delayed myelination, white matter hypoplasia, and "periventricular" leukomalacia: how are they related? Pediatr Neurol $15: 127-136$

3. Inder T, Huppi PS, Zientara GP, Maier SE, Jolesz FA, di Salvo D, Robertson R, Barnes PD, Volpe JJ 1999 Early detection of periventricular leukomalacia by diffusionweighted magnetic resonance imaging techniques. J Pediatr 134:631-634

4. Inder TE, Huppi PS, Warfield S, Kikinis R, Zientara GP, Barnes PD, Jolesz F, Volpe JJ 1999 Periventricular white matter injury in the premature infant is followed by reduced cerebral cortical gray matter volume at term. Ann Neurol 46:755-760

5. Huppi PS, Murphy B, Maier SE, Zientara GP, Inder TE, Barnes PD, Kikinis R, Jolesz FA, Volpe JJ 2001 Microstructural brain development after perinatal cerebral white matter injury assessed by diffusion tensor magnetic resonance imaging. Pediatrics 107:455-460

6. Towfighi J, Mauger D, Vannucci RC, Vannucci SJ 1997 Influence of age on the cerebral lesions in an immature rat model of cerebral hypoxia-ischemia: a light microscopic study. Brain Res Dev Brain Res 100:149-160

7. Hagberg H, Peebles D, Mallard C 2002 Models of white matter injury: comparison of infectious, hypoxic-ischemic, and excitotoxic insults. Ment Retard Dev Disabil Res Rev 8:30-38

8. Craig A, Ling Luo N, Beardsley DJ, Wingate-Pearse N, Walker DW, Hohimer AR, Back SA 2003 Quantitative analysis of perinatal rodent oligodendrocyte lineage progression and its correlation with human. Exp Neurol 181:231-240

9. Clancy B, Darlington RB, Finlay BL 2001 Translating developmental time across mammalian species. Neuroscience 105:7-17

10. Sizonenko SV, Sirimanne E, Mayall Y, Gluckman PD, Inder T, Williams CE 2003 Selective cortical alteration after hypoxic-ischemic injury in the very immature rat brain. Pediatr Res 54:263-269

11. Schmued LC, Hopkins KJ 2000 Fluoro-jade B: a high affinity fluorescent marker for the localization of neuronal degeneration. Brain Res 874:123-130

12. Koo EH, Sisodia SS, Archer DR, Martin LJ, Weidemann A, Beyreuther K, Fischer P, Masters CL, Price DL 1990 Precursor of amyloid protein in Alzheimer disease undergoes fast anterograde axonal transport. Proc Natl Acad Sci USA 87:1561-1565

13. Otsuka N, Tomonaga M, Ikeda K 1991 Rapid appearance of beta-amyloid precursor protein immunoreactivity in damaged axons and reactive glial cells in rat brain following needle stab injury. Brain Res 568:335-338

14. Stephenson DT, Rash K, Clemens JA 1992 Amyloid precursor protein accumulates in regions of neurodegeneration following focal cerebral ischemia in the rat. Brain Res 593:128-135

15. Nakamura Y, Takeda M, Niigawa H, Hariguchi S, Nishimura T 1992 Amyloid beta-protein precursor deposition in rat hippocampus lesioned by ibotenic acid injection. Neurosci Lett 136:95-98 
16. Streit WJ 1990 An improved staining method for rat microglial cells using the lectin from Griffonia simplicifolia (GSA I-B4). J Histochem Cytochem 38:1683-1686

17. Orlowski D, Soltys Z, Janeczko K 2003 Morphological development of microglia in the postnatal rat brain. A quantitative study. Int J Dev Neurosci 21:445-450

18. Milligan CE, Cunningham TJ, Levitt P 1991 Differential immunochemical marker reveal the normal distribution of brain macrophages and microglia in the developing rat brain. J Comp Neurol 314:125-135

19. Ivacko JA, Sun R, Silverstein FS 1996 Hypoxic-ischemic brain injury induces an acute microglial reaction in perinatal rats. Pediatr Res 39:39-47

20. Dommergues MA, Plaisant F, Verney C, Gressens P 2003 Early microglial activation following neonatal excitotoxic brain damage in mice: a potential target for neuroprotection. Neuroscience 121:619-628

21. Benjelloun N, Renolleau S, Represa A, Ben-Ari Y, Charriaut-Marlangue C 1999 Inflammatory responses in the cerebral cortex after ischemia in the P7 neonatal Rat. Stroke 30:1916-1923; discussion 1923-1914

22. Milligan CE, Levitt P, Cunningham TJ 1991 Brain macrophages and microglia respond differently to lesions of the developing and adult visual system. J Comp Neurol 314:136-146

23. Sizonenko SV, Sirimanne ES, Williams CE, Gluckman PD 2001 Neuroprotective effects of the N-terminal tripeptide of IGF-1, glycine-proline-glutamate, in the immature rat brain after hypoxic-ischemic injury. Brain Res 922:42-50

24. Northington FJ, Ferriero DM, Graham EM, Traystman RJ, Martin LJ 2001 Early neurodegeneration after hypoxia-ischemia in neonatal rat is necrosis while delayed neuronal death is apoptosis. Neurobiol Dis 8:207-219

25. Inder TE, Wells SJ, Mogridge NB, Spencer C, Volpe JJ 2003 Defining the nature of the cerebral abnormalities in the premature infant: a qualitative magnetic resonance imaging study. J Pediatr 143:171-179

26. Rice JE 3rd, Vannucci RC, Brierley JB 1981 The influence of immaturity on hypoxic-ischemic brain damage in the rat. Ann Neurol 9:131-141

27. Mallard EC, Williams CE, Johnston BM, Gluckman PD 1994 Increased vulnerability to neuronal damage after umbilical cord occlusion in fetal sheep with advancing gestation. Am J Obstet Gynecol 170:206-214

28. Barks JD, Silverstein FS 1992 Excitatory amino acids contribute to the pathogenesis of perinatal hypoxic-ischemic brain injury. Brain Pathol 2:235-243

29. Marret S, Mukendi R, Gadisseux JF, Gressens P, Evrard P 1995 Effect of ibotenate on brain development: an excitotoxic mouse model of microgyria and posthypoxiclike lesions. J Neuropathol Exp Neurol 54:358-370

30. Ferriero DM 2001 Oxidant mechanisms in neonatal hypoxia-ischemia. Dev Neurosci 23:198-202

31. Takashima S, Tanaka K 1978 Development of cerebrovascular architecture and its relationship to periventricular leukomalacia. Arch Neurol 35:11-16

32. McQuillen PS, Sheldon RA, Shatz CJ, Ferriero DM 2003 Selective vulnerability of subplate neurons after early neonatal hypoxia-ischemia. J Neurosci 23:3308-3315
33. Del Rio JA, Martinez A, Auladell C, Soriano E 2000 Developmental history of the subplate and developing white matter in the murine neocortex. Neuronal organization and relationship with the main afferent systems at embryonic and perinatal stages. Cereb Cortex 10:784-801

34. Clancy B, Cauller LJ 1999 Widespread projections from subgriseal neurons (layer VII) to layer I in adult rat cortex. J Comp Neurol 407:275-286

35. Vandevelde IL, Duckworth E, Reep RL 1996 Layer VII and the gray matter trajectories of corticocortical axons in rats. Anat Embryol (Berl) 194:581-593

36. Deguchi K, Oguchi K, Takashima S 1997 Characteristic neuropathology of leukomalacia in extremely low birth weight infants. Pediatr Neurol 16:296-300

37. Banker BQ, Larroche JC 1962 Periventricular leukomalacia of infancy. Arch Neurol $7: 386-410$

38. Meng SZ, Arai Y, Deguchi K, Takashima S 1997 Early detection of axonal and neuronal lesions in prenatal-onset periventricular leukomalacia. Brain Dev 19:480484

39. Burtrum D, Silverstein FS 1994 Hypoxic-ischemic brain injury stimulates glial fibrillary acidic protein mRNA and protein expression in neonatal rats. Exp Neurol 126:112-118

40. Hirayama A, Okoshi Y, Hachiya Y, Ozawa Y, Ito M, Kida Y, Imai Y, Kohsaka S, Takashima S 2001 Early immunohistochemical detection of axonal damage and glial activation in extremely immature brains with periventricular leukomalacia. Clin Neuropathol 20:87-91

41. Ohno M, Aotani H, Shimada M 1995 Glial responses to hypoxic/ischemic encephalopathy in neonatal rat cerebrum. Brain Res Dev Brain Res 84:294-298

42. Boya J, Calvo JL, Carbonell AL, Borregon A 1991 A lectin histochemistry study on the development of rat microglial cells. J Anat 175:229-236

43. Dammann O, Hagberg H, Leviton A 2001 Is periventricular leukomalacia an axonopathy as well as an oligopathy? Pediatr Res 49:453-457

44. Back SA, Han BH, Luo NL, Chricton CA, Xanthoudakis S, Tam J, Arvin KL, Holtzman DM 2002 Selective vulnerability of late oligodendrocyte progenitors to hypoxia-ischemia. J Neurosci 22:455-463

45. Back SA, Luo NL, Borenstein NS, Levine JM, Volpe JJ, Kinney HC 2001 Late oligodendrocyte progenitors coincide with the developmental window of vulnerability for human perinatal white matter injury. J Neurosci 21:1302-1312

46. Sizonenko SV, Kiss JZ 2004 Oligodendrocyte Progenitors do not die after hypoxiaischemia in the immature P3 rat brain. Pediatr Res 55:26A(146)

47. Cameron RS, Rakic P 1991 Glial cell lineage in the cerebral cortex: a review and synthesis. Glia 4:124-137

48. Deguchi K, Oguchi K, Matsuura N, Armstrong DD, Takashima S 1999 Periventricular leukomalacia: relation to gestational age and axonal injury. Pediatr Neurol 20:370-374 